\title{
Increasing the Performance of the Coupled-Dipole Approximation: A Spectral Approach
}

\author{
Nicolas B. Piller, Student Member, IEEE, and Olivier J. F. Martin
}

\begin{abstract}
We show that it is possible to increase the performance of the coupled-dipole approximation (CDA) for scattering by using concepts from the sampling theory. In standard CDA, the source in each discretized cell is represented by a point dipole and the corresponding scattered field given by Green's tensor. In the present approach, the source has a certain spatial extension, and the corresponding Green's tensor must be redefined. We derive these so-called filtered Green's tensors for one-dimensional (1-D), two-dimensional (2-D), and three-dimensional (3-D) systems, which forms the basis of our new scheme: the filtered coupled-dipole technique (FCD).

By reducing the aliasing phenomena related to the discretization of the scatterer, we obtain with FCD a more accurate description of the original scatterer.

The convergence and accuracy of FCD is assessed for 1-D, 2$D$, and 3-D systems and compared to CDA results. In particular we show that, for a given discretization grid, the scattering cross section obtained with FCD is more accurate (to a factor of 100). Furthermore, the computational effort required by FCD is similar to that of CDA.
\end{abstract}

Index Terms-Computation theory, convergence of numerical methods, diffraction, electromagnetic scattering, filtering, numerical analysis, propagation, sampling methods, signal processing.

\section{INTRODUCTION}

$\mathbf{T}$ HE coupled-dipole approximation (CDA) was first applied by Purcell and Pennypacker in 1973 to study the scattering by interstellar dust particles [1]. Later, the unfulfillment of the optical theorem by Purcell and Pennypacker's algorithm was noticed by several authors [2], [3]. This limitation was caused by the fact that in the series of dipole that represented a macroscopic object the self-interaction of each dipole was neglected. Nevertheless, Purcell and Pennypacker's algorithm was valid, but it required an extremely fine discretization of the object; furthermore, the optical theorem could not be used to compute the total scattering cross section. Some solutions were proposed to overcome this problem [2], [4]. In particular, Goedecke developed a pure macroscopical CDA theory [3]. The CDA was also expanded to anisotropic [5] and bianisotropic [6] materials.

The computation of the scattered field with CDA involves the solution of a large system of equations. This system of equations can be ill-conditioned when the scatterer is large. Therefore, primitive algorithms like LU factorization, which work only when the matrix is well conditioned, cannot

Manuscript received July 21, 1997. This work was supported by the Swiss National Science Foundation.

The authors are with the Laboratory of Field Theory and Microwave Electronics, Swiss Federal Institute of Technology, ETH-Zentrum, Zurich, 8092 Switzerland.

Publisher Item Identifier S 0018-926X(98)06094-3. be used for these problems. A more robust solver such as Givens factorization is required. Unfortunately, such solvers are generally more computation consuming.

An alternative has been described by Martin et al. [7] to compute the solution iteratively. This technique enables the rapid computation of a series of geometrically similar problems, because the solution of a previous problem may be reused as the starting point for another.

Recently, another method has been introduced to solve CDA equation systems [8], [9]. Based on the conjugate gradient algorithm [10] coupled with a fast Fourier transform (CGFFT), this technique provides a huge reduction in memory and computation requirements as they become proportional to $N$, respectively, $N \log (N)$, where $N$ is the number of volume discretization cells. The only restriction of CGFFT is that the discretization grid must be regular.

Later, the equivalence between CDA and the method of moment (MoM) for dielectric scatterers was evidenced [11]. Like this the fast multipole method (FMM) that was originally developed for MoM can be used directly to reduce the requirements when a regular discretization is not feasible [12]. Because of the reduction of accuracy associated with FMM [12], CGFFT should be chosen even when a regular discretization is possible [13].

In this paper, we demonstrate that the performance of the CDA can be improved by taking the sampling theory into account. First, in Section II, we recall the basis equations of CDA, as well as its traditional numerical implementation. The sampling theory is summarized in Section III. Because this theory needs some information on the spectrum ${ }^{1}$ of the functions to sample, the corresponding vectorial functions for electromagnetic fields are presented at the end of the section. In Section IV, the results of the two preceding sections are combined; the discretization of the volume integral equation is obtained using the sampling theory, which forms the modified CDA schemes presented in this paper. In Section V, numerical simulations are used to compare the different numerical schemes and study their convergence. Finally, our results are summarized in Section VI.

\section{The COUPLED-Dipole APPROXIMATION}

The aim of the CDA is to obtain the total vectorial electric field $\mathbf{E}(\mathbf{r})$ scattered by a three-dimensional (3-D) body $\epsilon_{0} \epsilon(\mathbf{r})$ illuminated by an incident field $\mathbf{E}^{\mathbf{O}}(\mathbf{r})$ and embedded in a homogeneous medium of permittivity $\epsilon_{0} \epsilon_{\text {ref }}$ and permeability

\footnotetext{
${ }^{1}$ The spectrum means the 1-D, 2-D, or 3-D Fourier transform of the spatial functions.
} 
$\mu_{0}$. The incident field has to fulfill the wave equation

$$
\nabla \times \nabla \times \mathbf{E}^{0}(\mathbf{r})-k_{0}^{2} \epsilon_{\mathrm{ref}} \mathbf{E}^{0}(\mathbf{r})=\mathbf{0}
$$

where the harmonic time dependence $\mathrm{e}^{-i \omega t}$ is assumed for each field function and $k_{0}$ is the wavenumber in vacuum: $k_{0}=\omega \sqrt{\mu_{0} \epsilon_{0}}$. The total field, which is the sum of the incident field and the scattered field, fulfills the wave equation for the inhomogeneous medium

$$
\nabla \times \nabla \times \mathbf{E}(\mathbf{r})-k_{0}^{2}\left(\epsilon_{\text {ref }}+\Delta \epsilon(\mathbf{r})\right) \mathbf{E}(\mathbf{r})=\mathbf{0}
$$

where $\Delta \epsilon(\mathbf{r})$ is defined as the dielectric contrast between the scatterer and the surrounding medium

$$
\Delta \epsilon(\mathbf{r})=\epsilon(\mathbf{r})-\epsilon_{\text {ref }}
$$

Let us note that $\Delta \epsilon(\mathbf{r})$ vanishes outside the scatterer.

\section{A. Solution of the Problem}

Evaluating the total field $\mathbf{E}(\mathbf{r})$ solution of (2) comes down to solving the volume integral equation

$$
\begin{aligned}
\mathbf{E}(\mathbf{r})= & \mathbf{E}^{0}(\mathbf{r})+k_{0}^{2} \lim _{\delta V \rightarrow 0} \int_{V^{\prime}-\delta V} \overleftrightarrow{G}\left(\mathbf{r}, \mathbf{r}^{\prime}\right) \\
& \cdot \Delta \epsilon\left(\mathrm{r}^{\prime}\right) \mathbf{E}\left(\mathrm{r}^{\prime}\right) \mathrm{d} \mathbf{r}^{\prime}-\stackrel{\leftrightarrow}{L} \cdot \frac{\Delta \epsilon(\mathbf{r})}{\epsilon_{\text {ref }}} \mathbf{E}(\mathbf{r})
\end{aligned}
$$

where $\overleftrightarrow{G}\left(\mathbf{r}, \mathbf{r}^{\prime}\right)$ is the dyadic Green's tensor of the reference medium. The integration volume $V^{\prime}$ includes all the domains where the function $\Delta \epsilon(\mathbf{r})$ does not vanish, and $\delta V$ is the exclusion volume: an infinitesimal domain around $\mathrm{r}$ to exclude the integration on the singularity of the dyadic Green's function for $\mathbf{r}=\mathbf{r}^{\prime}$. The source dyadic $\overleftrightarrow{L}$ depends only on the shape of the exclusion volume $\delta V$. Its value for usual shapes is tabulated in [14].

The volume integral equation (4) is valid for $\mathbf{r}$ both inside and outside the integration volume $V^{\prime}$ and represents the central equation for CDA.

\section{B. Traditional CDA}

The volume of the scatterer is decomposed into $N$ cells. In the traditional CDA, one assumes that each cell is small enough so that the variations of the total field $\mathbf{E}(\mathbf{r})$ and of the dielectric function $\Delta \epsilon(\mathbf{r})$ are negligible within the cell. Introducing $V_{i}$ and $\mathrm{r}_{i}$ for the volume, respectively, the position of the center of cell $i$, one can then define

$$
\begin{gathered}
\left.\mathbf{E}(\mathbf{r})\right|_{\mathbf{r} \in V_{i}} \approx \mathbf{E}_{i}=\mathbf{E}\left(\mathbf{r}_{i}\right) \\
\left.\Delta \epsilon_{i}(\mathbf{r})\right|_{\mathbf{r} \in V_{i}} \approx \Delta \epsilon_{i}=\Delta \epsilon\left(\mathbf{r}_{i}\right) .
\end{gathered}
$$

Using (5) and (6), the integral equation (4) is decomposed into a sum of integrals over each cell. This gives for $\mathbf{r}=\mathbf{r}_{i}$

$$
\begin{aligned}
\mathbf{E}_{i}= & \mathbf{E}^{0}\left(\mathbf{r}_{i}\right)+k_{0}^{2} \sum_{k=1, k \neq i}^{N} \overleftrightarrow{G}\left(\mathbf{r}_{i}, \mathbf{r}_{k}\right) \cdot \Delta \epsilon_{k} \mathbf{E}_{k} V_{k} \\
& +k_{0}^{2} \lim _{\delta V \rightarrow 0} \int_{V_{i}-\delta V} \overleftrightarrow{G}\left(\mathbf{r}_{i}, \mathbf{r}^{\prime}\right) \mathrm{d} \mathbf{r}^{\prime} \\
& \cdot \Delta \epsilon_{i} \mathbf{E}_{i}-\overleftrightarrow{L} \cdot \frac{\Delta \epsilon_{i}}{\epsilon_{\mathrm{ref}}} \mathbf{E}_{i}
\end{aligned}
$$

where we have assumed that the variations of $\overleftrightarrow{G}\left(\mathbf{r}_{i}, \mathbf{r}^{\prime}\right)$ for $\mathbf{r}^{\prime}$ in the $k$ th cell can be neglected for $k \neq i$.

The integral in (7) is difficult to evaluate because $\overleftrightarrow{G}\left(\mathbf{r}_{i}, \mathbf{r}^{\prime}\right)$ varies extremely rapidly when $\mathbf{r}^{\prime}$ becomes close to $\mathbf{r}_{i}$. For convenience, let us represent this integral by $\vec{M}_{i}$ and call it the self-induction term. Different approximations for $\overleftrightarrow{M}_{i}$ have been given in the literature [2]-[4]. For example, Purcell and Pennypacker neglect this term in their calculation (i.e., $\overleftrightarrow{M}_{i}=$ $\overleftrightarrow{0}$ ) [1]. This is only possible for very fine sampling and does not allow the application of the optical theorem to evaluate the total scattering cross section [3]. To overcome these drawbacks, Draine proposed approximating each cell using a sphere [or a circle for two-dimensional (2-D) calculations] of similar volume [2]. The integral in (7) can then be performed analytically [15]. An interesting comparison of the different approximations for $\overleftrightarrow{M}_{i}$ is made by $\mathrm{Ku}$ [16].

Introducing the self-induction term $\overleftrightarrow{M}_{i}$ into (7) gives

$$
\begin{aligned}
\mathbf{E}_{i}= & \mathbf{E}^{0}\left(\mathbf{r}_{i}\right)+k_{0}^{2} \sum_{k=1, k \neq i}^{N} \overleftrightarrow{G}\left(\mathbf{r}_{i}, \mathbf{r}_{k}\right) \cdot \Delta \epsilon_{k} \mathbf{E}_{k} V_{k} \\
& +k_{0}^{2} \overleftrightarrow{M}_{i} \Delta \epsilon_{i} \mathbf{E}_{i}-\stackrel{\leftrightarrow}{L} \cdot \frac{\Delta \epsilon_{i}}{\epsilon_{\mathrm{ref}}} \mathbf{E}_{i} .
\end{aligned}
$$

Writing this last equation for each $i=1 \cdots N$ and for each vector component gives a large system of algebraic equations with $3 N$ equations and $3 N$ unknowns for $3-\mathrm{D}$ vectorial problems. The factorization of this system of equations gives $\mathbf{E}_{i}$, the electric field inside the volume $V^{\prime}$. To compute the field outside $V^{\prime}$, the integral equation (4) can be discretized for any $\mathbf{r} \notin V^{\prime}$ using again (5) and (6). This leads to

$$
\mathbf{E}(\mathbf{r})=\mathbf{E}^{0}(\mathbf{r})+k_{0}^{2} \sum_{k=1}^{N} \overleftrightarrow{G}\left(\mathbf{r}, \mathbf{r}_{k}\right) \cdot \Delta \epsilon_{k} \mathbf{E}_{k} V_{k} .
$$

Therefore, the computation of the field at a point $\mathbf{r}$ outside the scatterer does not require the solution of a system of equations, but can simply be obtained from the field inside the object.

Two different conditions have been implicitly introduced by the previous approximations. First, the variation of the electric field in a cell and the variation of the dyadic Green's tensor in a cell were neglected [except for the computation of $\overleftrightarrow{M}_{i}$, where the variations of $\overleftrightarrow{G}\left(\mathbf{r}, \mathbf{r}^{\prime}\right)$ over a cell were taken into account]. Second, the shape of each cell was approximated by a sphere to compute $\overleftrightarrow{M}_{i}$, thereby forcing the cell sizes in all the three directions to be more or less similar. In order to obtain accurate enough results, these two conditions require that the cell dimensions are much smaller than the wavelength, which leads to a large system of equations and therefore increases the memory and computation requirements.

Finally, let us note that the scattering by an object embedded in a particular inhomogeneous medium (surface, multilayered substrate) is easily computed with the CDA if the Green's tensor is known for this particular medium. In this case, only the scatterer has to be modeled with dipoles, leading to a huge reduction in computation time and memory requirements [17]. 


\section{The SAMPLing THEORY APPLIED TO ELECTROMAGNETIC FIELDS}

In the previous section, the CDA technique was presented. In this paper, we propose improvement of this technique by using the sampling theory to decompose the volume integral equation (4). The sampling theory is based on the knowledge of the different spectral domains of a given function; in particular on the knowledge of the spectral domains that contain either important or negligible information. Thus, the spectrum lying in important domains must be taken into account, while remaining area of the spectrum can be neglected to approximate the function with a given precision.

In this section, we first recall the sampling theory in a general way; then we study the spectrum of the electric field function and the most accurate way to represent this function by discrete samples.

\section{A. Sampling and Reconstructing a Function}

1) Sampling Theory for a 1-D Function: The continuous function $f(x)$ is represented by discrete values or samples. Then, an estimation of the original function is obtained using a reconstruction filter. As we will see, two kinds of distortions are introduced by this sampling/reconstruction operation, but it is possible to attenuate one of them by suppressing some spectral components of the original function prior to sampling, with a filter called antialiasing filter (AAF) [18], [19]. Let us call $\tilde{f}(x)$ the function obtained by filtering the original function with the antialiasing filter $H^{\mathrm{a}}(k)$. The spectrum $\tilde{F}(k)$ of $\tilde{f}(x)$ is

$$
\tilde{F}(k)=H^{\mathrm{a}}(k) F(k)
$$

where $F(k)$ is the spectrum of the original function $f(x)$. The function $\tilde{f}(x)$ is then sampled with a regular sampling distance $\Delta_{x}$. The sampled function $f^{\mathrm{d}}(x)$ can be represented with the help of Dirac delta functions $\delta(x)$

$$
f^{\mathrm{d}}(x)=\sum_{l} \tilde{f}_{l} \delta\left(x-l \Delta_{x}\right) \Delta_{x}
$$

where $\tilde{f}_{l}$ are the sample values $\tilde{f}\left(l \Delta_{x}\right)$. Let us emphasize that the sampling operation (11) corresponds to the numerical discretization procedure effectuated in a numerical scheme.

As the sampled function is fully determined by the continuous function $\tilde{f}(x)$, its spectrum $F^{\mathrm{d}}(k)$ is fully determined by $\tilde{F}(k)[20]$ :

$$
F^{\mathrm{d}}(k)=F(k)+\sum_{p \neq 0} F\left(k-p \Delta_{k}\right)
$$

where the sum in (12) represents the spectral repetitions produced by the sampling operation. Each spectral repetition is equivalent to the spectrum of the continuous function, shifted by a multiple of the sampling circular frequency $\Delta_{k}=2 \pi / \Delta_{x}$.

For example, the spectrum $F(k)$ of an original function is given in Fig. 1(a1). First, we use no antialiasing filter, i.e., $H^{\mathrm{a}}(k)=1$ and $\tilde{F}(k)=F(k)$. Sampling the function with a sampling rate $\Delta_{x}$ gives the spectrum in Fig. 1(a2). The
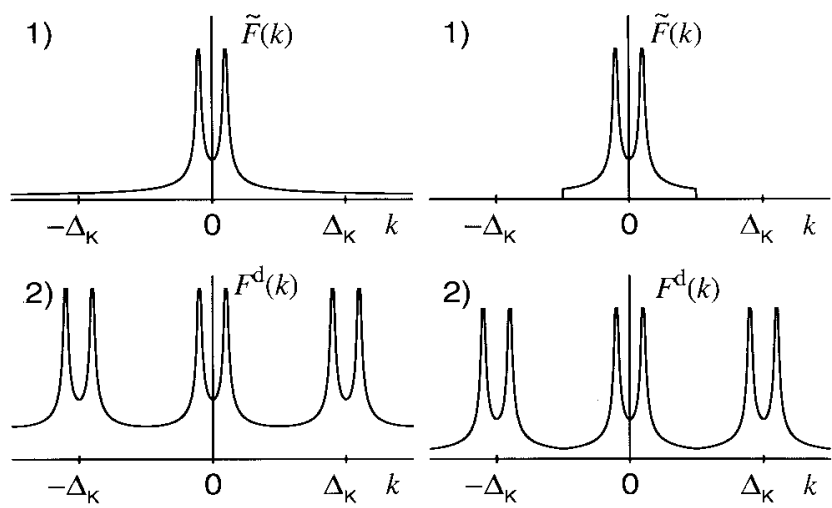

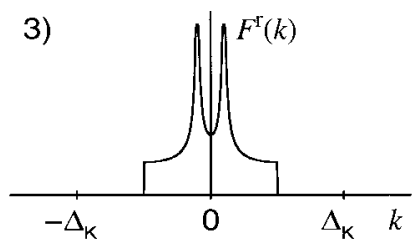

(a)

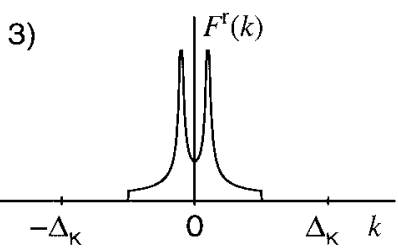

(b)
Fig. 1. Spectra of the 1-D function during the sampling and reconstruction operation: (a) without antialiasing filter and (b) with an ideal antialiasing filter. 1) Prior sampling; 2) sampled; and 3 ) after reconstruction.

superposition of the spectral repetitions with the spectrum of the original function is visible [Fig. 1(a2)].

Reconstructing the function, i.e., approximating the original function, reduces to extraction of the spectral domain where the spectrum of the original function is more important than the spectral repetitions. This operation is made with the reconstruction filter $H^{\mathrm{r}}(k)$. The spectrum of the reconstructed function becomes

$$
F^{\mathrm{r}}(k)=H^{\mathrm{r}}(k) F^{\mathrm{d}}(k) .
$$

For the example of Fig. 1, a well-adapted reconstruction filter is a low-pass with cutoff frequency equal to $\Delta_{k} / 2$. The spectrum of the signal reconstructed with this filter is given in Fig. 1(a3).

Two kinds of distortions are introduced by the sampling operation. First, when the original function has nonzero spectrum falling in the blocking region of the reconstruction filter, this part of the spectrum is lost, producing linear distortions. Comparing the original [Fig. 1(a1)] and reconstructed [Fig. 1(a3)] function spectra, this distortion is noticeable for circular frequencies $|k|>\Delta_{k} / 2$, where the spectrum of the original function is lost.

The second kind of distortion appears when nonzero spectral repetitions lie in a nonblocking region of the reconstruction filter. In this case, spectrum parts that do not exist in the original function may appear in the reconstructed function, producing nonlinear distortions, called aliasing. This is noticeable in Fig. 1(a3) for $|k|<\Delta_{k} / 2$, where the spectrum is modified.

Nonlinear distortions can be suppressed by using a better antialiasing filter. This antialiasing filter must remove the spectral components of the continuous signal that produce nonzero spectral repetitions in the pass-through domain of the reconstruction filter. A low-pass filter with a circular 
cutoff frequency $\Delta_{k} / 2$ would be optimal. Using such an antialiasing filter on the spectrum $F(k)$ in Fig. 1(a1) gives the spectrum $\tilde{F}(k)$ in Fig. 1(b1). Sampling this function does not produce spectrum overlapping [Fig. 1(b2)], and then the reconstructed function spectrum is only spoiled by linear distortions [Fig. 1(b3)]. The reconstructed spectrum $F^{\mathrm{r}}(k)$ is exactly equal to $\tilde{F}(k)$. This is possible because in our example $\tilde{F}(k)$ fulfills the sampling theorem [21], [22].

The reconstructed function $f^{\mathrm{r}}(x)$ can be determined by inverse Fourier transform of its spectrum $F^{\mathrm{r}}(k)$, or by direct convolution of the sampled function with the filter impulse response $h^{\mathrm{r}}(x)$. Using the properties of the Dirac delta function, this leads to

$$
f^{\mathrm{r}}(x)=\sum_{l} f_{l} h^{\mathrm{r}}\left(x-l \Delta_{x}\right) \Delta_{x}
$$

where $h^{\mathrm{r}}(x)$ is evaluated by inverse Fourier transform of its spectrum $H^{\mathrm{r}}(k)$. In our example, the filter is a low-pass with cutoff frequency $\Delta_{k} / 2$; its impulse response is

$$
h^{\mathrm{r}}(x)=\frac{\sin \left(\pi x / \Delta_{x}\right)}{\pi x} .
$$

As the spectrum components with $|k|>\Delta_{k} / 2$ are lost by the sampling/reconstruction operation, the sampling rate must be large enough to preserve the important part or the spectrum.

2) Sampling 2-D and 3-D Field Functions: The sampling theory is generally used for scalar functions. But in this paper, the function to sample and reconstruct is a vectorial field function and little literature exists for such a case. Nevertheless, each Cartesian component of the field function can be considered as a scalar function, which relates our problem to the traditional sampling theory and in the remaining of the paper we will use "spectrum" for the "spectrum of each field component."

The vectorial 2-D or 3-D function is sampled on a regular grid, with step lengths $\Delta_{u}$ in the $\hat{u}$ direction, corresponding to a sampling circular frequency $\Delta_{k_{u}}=2 \pi / \Delta_{u}$ for this direction ( $u=x, y$ for 2-D and $u=x, y, z$ for 3-D).

Again, the optimal anti-aliasing filter is a low-pass with cutoff circular frequency $\Delta_{k_{u}} / 2$ in the spectral direction $\hat{k}_{u}$. For example, the spectrum of an original 2-D function $\mathbf{F}(\mathbf{k})$ is plotted in Fig. 2(a). After suppression of the high spectral components by this optimal filter, the spectrum $\tilde{\mathbf{F}}(\mathbf{k})$ is plotted in Fig. 2(b).

As in the 1-D case, the spectrum of the sampled function is formed by the original spectrum plus the spectral repetitions [Fig. 2(c)], the latter showing a shift $\Delta_{k_{u}}$ in each $\hat{k}_{u}$ direction [23].

Using a low-pass reconstruction filter with cutoff circular frequency $\Delta_{k_{u}}$ in the spectral direction $\hat{k}_{u}$ (i.e., a square pass-through region) the reconstructed spectrum is perfectly identical to $\tilde{\mathbf{F}}(\mathbf{k})$ [Fig. 2(b)].

As we will see in Section IV, the reconstructed function has to be integrated after its multiplication with Green's tensor. While the impulse response is best expressed in Cartesian coordinates, Green's tensor is best expressed in circular (2D) or spherical (3-D) coordinates. The integration would be easier if these two product elements had the same coordinate

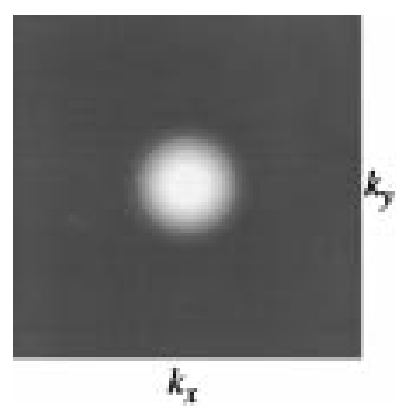

(a)

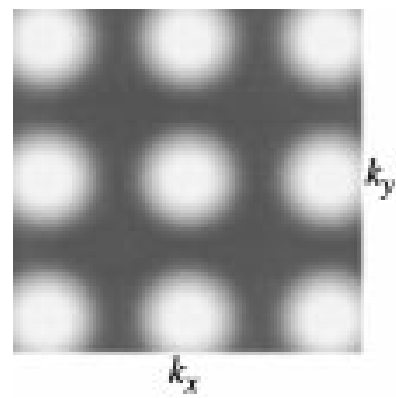

(c)

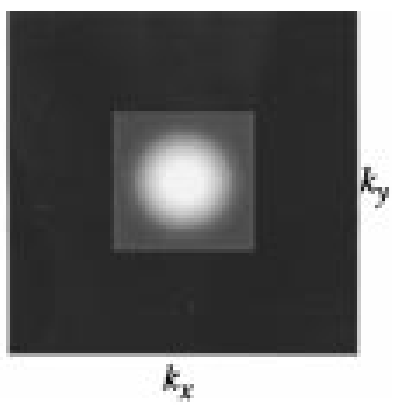

(b)

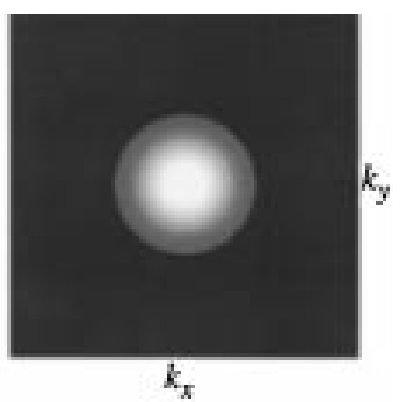

(d)
Fig. 2. Influence of the reconstruction filter on the spectra of a 2-D function: (a) original function, (b) filtered with anti-aliasing filter, (c) filtered and sampled, and (d) reconstructed with a circular reconstruction filter. If an ideal reconstruction filter were used, the reconstructed spectrum would coincide with spectrum (b).

system. A work-around consists in restricting the pass-through domain of the reconstruction filter to a circle (or a sphere for 3-D) included in the pass-through region of the square reconstruction filter. The spectrum of the signal reconstructed with such a filter is plotted in Fig. 2(d). Some of the spectrum information contained in the edges of the square is lost, as can be noticed in Fig. 2(d). We have verified with numerical simulations that the influence of these additional linear distortions on the solution of our algorithm is negligible. Therefore, it is possible to take advantage of this reduction of the pass-through domain to make the integration of the product of the impulse response with Green's tensor easier. The spectral domain $|\mathrm{k}|>\min _{u}\left(\Delta_{k_{u}}\right) / 2$ is then lost by this sampling/reconstruction operation. Therefore, the sampling rate $\Delta_{k_{u}}$ for each $\hat{u}$ direction must be chosen large enough to preserve the important part of the spectrum.

Defining $\mathrm{r}_{i}$ as the position of the $i$ th sample, the corresponding sample value $\tilde{\mathbf{f}}_{i}$ becomes

$$
\tilde{\mathbf{f}}_{i}=\tilde{\mathbf{f}}\left(\mathrm{r}_{i}\right) .
$$

As in the 1-D case (14), the reconstructed function $\mathrm{f}^{\mathrm{r}}(\mathrm{r})$ is obtained by filtering the sampled signal with the reconstruction filter $h^{\mathrm{r}}(\mathbf{r})$, and is used to approximate the original function

$$
\mathbf{f}(\mathbf{r}) \approx \mathbf{f}^{\mathrm{r}}(\mathrm{r})=\sum_{i} \tilde{\mathbf{f}}_{i} h^{\mathrm{r}}\left(\mathrm{r}-\mathbf{r}_{i}\right) V
$$

where $V$ is the volume of the cell, equal to $\Delta_{x} \Delta_{y}$ for the 2-D case and to $\Delta_{x} \Delta_{y} \Delta_{z}$ for the 3-D case. 


\section{B. Spectrum of the Electric Field Function}

Our objective is to use the sampling theory to obtain an approximation for the integrand of (4). Therefore, we will first study the spectrum of the product $\mathbf{E}(\mathbf{r}) \Delta \epsilon(\mathbf{r})$. Actually we first study the spectra of $\mathbf{E}(\mathbf{r})$ and $\Delta \epsilon(\mathbf{r})$ separately and then deduce information on the spectrum of their product. Then we will propose a way to suppress the high spectral components of this product, in order to reduce aliasing by the sampling operation.

We consider a piecewise homogeneous scatterer that can be decomposed into a finite number $D$ of closed homogeneous regions $V_{n}$. Each region $V_{n}$ has a wave number $k_{\mathrm{d}_{n}}$ and a dielectric function $\Delta \epsilon(\mathbf{r})$ equal to $\Delta \epsilon_{n}$. Assuming that $\Delta \epsilon_{n}(\mathbf{r})$ vanishes outside $V_{n}$, the function product $\mathbf{E}(\mathbf{r}) \Delta \epsilon(\mathbf{r})$ can be rewritten as a sum

$$
\mathbf{E}(\mathbf{r}) \Delta \epsilon(\mathbf{r})=\sum_{n=1}^{D} \mathbf{E}_{n}(\mathbf{r}) \Delta \epsilon_{n}(\mathbf{r})
$$

where $\Delta \epsilon_{n}(\mathbf{r})$ and $\mathbf{E}_{n}(\mathbf{r})$ are equal to $\Delta \epsilon_{n}$, respectively, $\mathbf{E}(\mathbf{r})$, inside $V_{n}$. Outside $V_{n}$, as $\Delta \epsilon_{n}(\mathbf{r})$ vanishes, $\mathbf{E}_{n}(\mathbf{r})$ can take arbitrary values.

Inside the region $V_{n}$, we use the fact that the field $\mathbf{E}_{n}(\mathbf{r})$ can always be approximated by the series expansion

$$
\mathbf{E}_{n}(\mathbf{r})=\sum_{i=1}^{N} a_{i} \mathbf{g}_{i}(\mathbf{r}) .
$$

For a 1-D problem, the field can be exactly represented in a homogeneous region with a sum of only two functions, representing plane waves propagating in the positive and negative directions [24]. For the 2-D and 3-D cases, the field can only be approximated, but with an arbitrary precision, with a finite number of expansion functions. An appropriate approximation basis $\mathbf{g}_{i}(\mathbf{r})$ is the regular basis also called Bessel multipoles. (See [25, Sec. C.2], [26] for 2-D and [25, Sec. C.3], [27] for 3-D.) This defines a "complete" basis and can therefore also represent evanescent waves on a finite domain.

Let us define $\mathcal{D}_{0}$ as the spectral domain where the spectrum of the field functions $\mathbf{g}_{i}(\mathbf{r})$ does not vanish. For the abovementioned function $\mathrm{g}_{i}(\mathbf{r}), \mathcal{D}_{0}$ is only formed by the two discrete points $\mp k_{\mathrm{d}_{2}}$ for 1-D, by a circle of radius $k_{\mathrm{d}_{2 n}}$ for 2-D, ${ }^{2}$ and by a sphere of radius $k_{\mathrm{d}_{n}}$ for 3-D. Because of the linearity of the Fourier transform, the spectrum of any linear combination of these functions, and therefore also the spectrum of the field approximation (19) is nonzero only in $\mathcal{D}_{0}$.

The spectrum of the dielectric function in the domain $V_{n}$ can be evaluated by Fourier transform. Therefore, we can use some properties of the Fourier transform to obtain information on the spectrum of $\Delta \epsilon_{n}(\mathbf{r})$. First, because $\Delta \epsilon_{n}(\mathbf{r})$ is constant on $V_{n}$, the maximum value of this spectrum is at the point $\mathbf{k}=\mathbf{0}$. Second, because $\Delta \epsilon_{n}(\mathbf{r})$ does not vanish in a closed domain, the spectral domain where the spectrum does not vanish is not closed. Third, the uncertainty principle bounds

\footnotetext{
${ }^{2}$ To simplify the notation, we assume no propagation in $\hat{z}$ direction. In a general case, the circle radius is equal to the transversal wave number in the concerned domain $\kappa_{\mathrm{d}_{n}}$.
}

the relation between the domain size $V_{n}$ and the spectral domain size containing information: when the domain $V_{n}$ is small, the spectral information is more spread than when $V_{n}$ is large. Finally, the spectrum of $\Delta \epsilon_{n}(\mathbf{r})$ is more spread when the boundary of $V_{n}$ is complicated, because higher frequency spectral components are needed to represent this boundary accurately.

Consequently, the spectrum of each Cartesian component of the product $\mathbf{E}_{n}(\mathbf{r}) \Delta \epsilon_{n}(\mathbf{r})$ being the convolution of their spectra, the following deductions can be done. First, the spectral domain on and at the vicinity of $\mathcal{D}_{0}$ contains important information. Second, when $V_{n}$ is small or has a complicated boundary, a larger neighborhood of $\mathcal{D}_{0}$ contains important information in the spectral domain.

Because of the linearity of the Fourier transform, the spectrum of the function $\Delta \epsilon(\mathbf{r}) \mathbf{E}(\mathbf{r})$ for the complete scatterer is equal to the sum of the spectra $\Delta \epsilon_{n}(\mathbf{r}) \mathbf{E}_{n}(\mathbf{r})$ associated with the different homogeneous regions. Therefore, the spectral domain containing important information for the total function must include all the domains containing important information for each function $\Delta \epsilon_{n}(\mathbf{r}) \mathbf{E}_{n}(\mathbf{r})$.

Taking into account the results of Section III-A2, we see that the sampling rate $\Delta_{u}$ has to be larger than $2 k_{\mathrm{d}_{n}}$. This means that we must sample with at least two points per wavelength; otherwise a part of $\mathcal{D}_{0}$ will be lost, leading to an extremely bad approximation of the field. This minimal sampling rate must be raised when the different domains are small or have complex boundaries. Furthermore, it is obvious that increasing the sampling rate produces a better approximation: a larger part of the spectrum being then taken into account.

As a 2-D spectrum example, let us consider the field scattered by an homogeneous infinite cylinder. We assume that the electric field $\mathbf{E}_{n}(\mathbf{r})$ is limited to the first expansion function $J_{0}\left(k_{\mathrm{d}} \sqrt{x^{2}+y^{2}}\right) \hat{z}$. The corresponding spectrum is reported in Fig. 3(a1). The domain where the spectrum does not vanish, a circle of radius $k_{\mathrm{d}}$, is clearly visible. The spectrum of the associated dielectric function $\Delta \epsilon_{n}(\mathbf{r})$, which is a cylinder of small radius, is represented in Fig. 3(a2). The spectrum of the product $\Delta \epsilon_{n}(\mathbf{r}) \mathbf{E}_{n}(\mathbf{r})$ has its maxima on and near $\mathcal{D}_{0}$ [Fig. 3(a3)]. As the spectrum of the dielectric function [Fig. 3(a2)], the spectrum of this product decreases at high frequencies, but never vanishes [Fig. 3(a3)].

As we have seen in Section III-A2, the high spectral components of the function to sample must be suppressed to eliminate aliasing. Because the electric field is a priori unknown, it is not possible to directly filter the product $\Delta \epsilon_{n}(\mathbf{r}) \mathbf{E}_{n}(\mathbf{r})$. It is nevertheless possible to suppress its high spectrum components by filtering only $\Delta \epsilon_{n}(\mathbf{r})$. Using a low-pass filter with a cutoff circular frequency $k_{u}^{\mathrm{a}}$ in the $\hat{k}_{u}$ direction, the resulting function, which we define as $\Delta \tilde{\epsilon}_{n}(\mathbf{r})$, has its spectrum represented in Fig. 3(b2). The spectrum of the product $\Delta \tilde{\epsilon}_{n}(\mathbf{r}) \mathbf{E}_{n}(\mathbf{r})$ is given in Fig. 3(b3). This spectrum being the convolution of the spectrum of $\mathbf{E}_{n}(\mathbf{r})$ [Fig. 3(b1)] and the spectrum of $\Delta \tilde{\epsilon}_{n}(\mathbf{r})$ [Fig. 3(b2)], it vanishes for $\left|k_{u}\right|>k_{u}^{\mathrm{a}}+$ $k_{\mathrm{d}_{n 2}}$. To suppress any aliasing, the cutoff circular frequency $k_{u}^{\mathrm{a}}$ must satisfy

$$
k_{u}^{\mathrm{a}}+k_{\mathrm{d}_{n}} \leq \Delta_{k_{u}} / 2 .
$$


1)

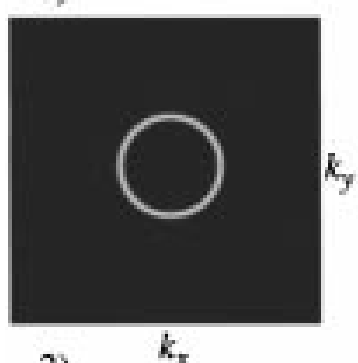

2)

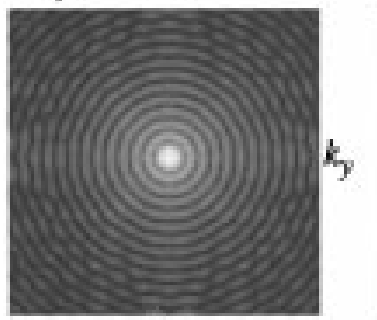

3)

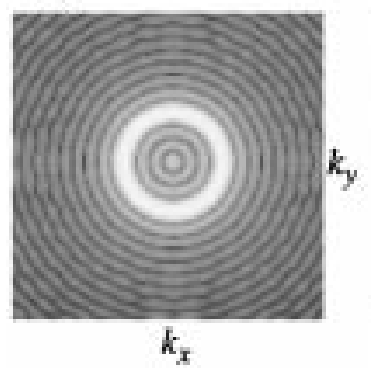

(a)
1)

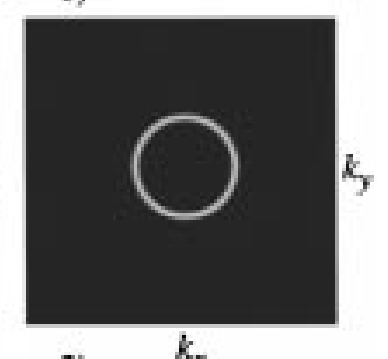

2)
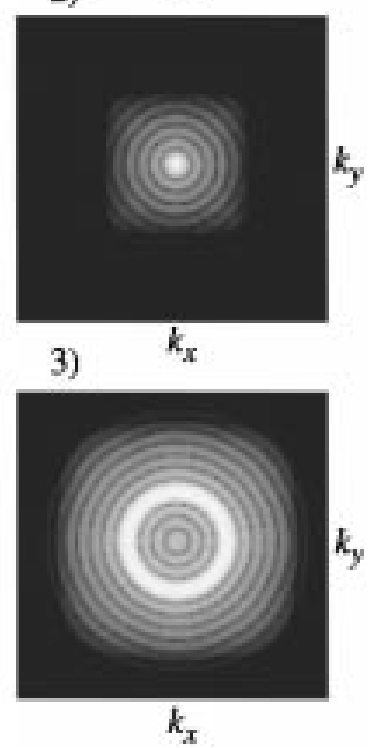

(b)
Fig. 3. Limitation of the spectral domain of $\Delta \tilde{\epsilon}(\mathbf{r}) \mathbf{E}(\mathbf{r})$ using a filter on $\Delta \epsilon(\mathbf{r})$ : (a) without low pass and (b) with low pass. 1) Spectrum of $\mathbf{E}(\mathbf{r}) .2$ ) Spectrum of $\Delta \tilde{\epsilon}(\mathbf{r}) ; 3)$ Spectrum of $\Delta \tilde{\epsilon}(\mathbf{r}) \mathbf{E}(\mathbf{r})$.

The boundary (20) seems nevertheless too restrictive, because such a low-pass characteristic suppresses all the nonlinear distortions but produces a lot of linear distortions. Increasing $k_{u}^{\mathrm{a}}$ introduces aliasing but reduces the linear distortions. Using numerical simulations, we obtained an optimum for

$$
k_{u}^{\mathrm{a}}=\Delta_{k_{u}} / 2 .
$$

The use of an ideal low-pass filter to obtain $\Delta \tilde{\epsilon}(\mathbf{r})$ is impossible, because such a filter has an infinite impulse response (IIR), making the nonvanishing domain of $\Delta \tilde{\epsilon}(\mathbf{r})$ infinite and the domain to sample infinite as well. Therefore, we must use a finite impulse response filter (FIR). We choose to use the windowing technique [28, Ch. 3.8] with an Hanning window (Hanning-FIR) [28, Ch. 3.10] of size $L_{u}=3 \Delta_{x}$.

Analytically filtering a dielectric function with such a continuous filter is generally impossible. To evaluate $\Delta \tilde{\epsilon}(\mathbf{r})$, the filter must be discretized, i.e., the impulse response and the dielectric constant are sampled and then convoluted. The sampling distance used for this operation is $\Delta_{u} / 8$.

Defining $\Delta \tilde{\epsilon}_{i}=\Delta \tilde{\epsilon}\left(\mathbf{r}_{i}\right), \mathbf{E}_{i}=\mathbf{E}\left(\mathbf{r}_{i}\right)$ and using (17), we obtain an approximation for the original function:

$$
\mathbf{E}(\mathbf{r}) \Delta \epsilon(\mathbf{r}) \approx \sum_{i} \Delta \tilde{\epsilon}_{i} \mathbf{E}_{i} h^{\mathrm{r}}\left(\mathbf{r}-\mathbf{r}_{i}\right) V
$$

Finding an optimal filter for the dielectric function is difficult and the proposed FIR filter is only one possible choice from many. Another choice is a mean-filter, where $\Delta \tilde{\epsilon}_{i}$ becomes equal to the mean value of $\Delta \epsilon(\mathbf{r})$ over mesh $V_{i}$. This last filter is easy to implement and gives relatively good results, as will be seen in Section V. Nevertheless, an optimization study for determining the optimal filter remains to be made.

\section{Coupled-Dipole APPROXimation BASED ON SAMPLING THEORY}

In Sections II and III-A, the electromagnetic integral equation and the signal reconstruction were presented. Now, the results of these two theories are combined to formulate a new CDA scheme.

The approximation (22) of the continuous function product $\mathbf{E}(\mathbf{r}) \Delta \epsilon(\mathbf{r})$ is introduced in (4):

$$
\begin{aligned}
\mathbf{E}(\mathbf{r})= & \mathbf{E}^{0}(\mathbf{r})+k_{0}^{2} \lim _{\delta V \rightarrow 0} \int_{V^{\prime}-\delta V} \overleftrightarrow{G}\left(\mathbf{r}, \mathbf{r}^{\prime}\right) \\
& \cdot \sum_{k} h^{\mathrm{r}}\left(\mathbf{r}^{\prime}-\mathbf{r}_{k}\right) \Delta \tilde{\epsilon}_{k} \mathbf{E}_{k} V \mathrm{~d} \mathbf{r}^{\prime}-\overleftrightarrow{L} \cdot \frac{\Delta \tilde{\epsilon}(\mathbf{r})}{\epsilon_{\mathrm{ref}}} \mathbf{E}(\mathbf{r})
\end{aligned}
$$

Interchanging the integration/summation order, (23) becomes

$$
\begin{aligned}
\mathbf{E}(\mathbf{r})= & \mathbf{E}^{0}(\mathbf{r})+k_{0}^{2} \sum_{k=1}^{N} \overleftrightarrow{G}^{\mathrm{F}}\left(\mathbf{r}, \mathbf{r}_{k}\right) \\
& \cdot \Delta \tilde{\epsilon}_{k} \mathbf{E}_{k} V-\overleftrightarrow{L} \cdot \frac{\Delta \tilde{\epsilon}(\mathbf{r})}{\epsilon_{\mathrm{ref}}} \mathbf{E}(\mathbf{r})
\end{aligned}
$$

where the tensor $\overleftrightarrow{G}^{\mathrm{F}}\left(\mathbf{r}, \mathbf{r}_{k}\right)$ is

$$
\stackrel{\leftrightarrow}{G}^{\mathrm{F}}\left(\mathbf{r}, \mathbf{r}_{k}\right)=\lim _{\delta V \rightarrow 0} \int_{V^{\prime}-\delta V} \overleftrightarrow{G}\left(\mathbf{r}, \mathbf{r}^{\prime}\right) h^{\mathrm{r}}\left(\mathbf{r}^{\prime}-\mathbf{r}_{k}\right) \mathrm{dr}^{\prime} .
$$

The integral in (25) is a convolution, which means that $\overleftrightarrow{G}^{\mathrm{F}}\left(\mathbf{r}, \mathbf{r}_{k}\right)$ is equal to the Green's tensor $\overleftrightarrow{G}\left(\mathbf{r}, \mathbf{r}_{k}\right)$ filtered by the reconstruction filter $h^{\mathrm{r}}(\mathbf{r})$. This filtered Green's tensor is developed for 1-D, 2-D, and 3-D in Appendix A. It is interesting to note that it does not have a singularity for $\mathbf{r}=\mathbf{r}_{k}$. as was the case for the nonfiltered Green's tensor. Equation (24) is valid for $\mathbf{r}$ inside and outside the scatterer domain. We rewrite this relation for $\mathbf{r}=\mathbf{r}_{i}$ to obtain a system of linear equations:

$$
\mathbf{E}_{i}=\mathbf{E}^{0}\left(\mathbf{r}_{i}\right)+k_{0}^{2} \sum_{k=1}^{N} \overleftrightarrow{G}^{\mathrm{F}}\left(\mathbf{r}_{i}, \mathbf{r}_{k}\right) \cdot \Delta \tilde{\epsilon}_{k} \mathbf{E}_{k} V-\overleftrightarrow{L} \cdot \frac{\Delta \tilde{\epsilon}(\mathbf{r})}{\epsilon_{\mathrm{ref}}} \mathbf{E}_{i} .
$$

This system of equations is quite similar to (18). The selfinduction term $M_{i}$ is now expressed by $\stackrel{\leftrightarrow}{G}^{\mathrm{F}}\left(\mathrm{r}_{i}, \mathbf{r}_{i}\right) V$, and the Green's tensor is replaced by the filtered Green's tensor. Therefore, we name this new scheme filtered coupled-dipole (FCD). The numerical solution of the problem is obtained in a similar way as before: first the system of (26) must be solved to obtain the field on the sampling points. Then the field outside the scatterer can be computed with (24). In addition, the field 
inside the scatterer but at different positions than the sampling points can also be computed with (24).

As for CDA, the analogy between FCD and MoM for dielectric scatterers can be remarked [11]. Indeed, writing FCD in the MoM formulation gives the reconstruction functions $h^{\mathrm{r}}\left(\mathbf{r}-\mathbf{r}_{k}\right)$ as basis functions and the Dirac delta functions $\delta\left(\mathbf{r}-\mathbf{r}_{i}\right)$ as test functions. MoM generally uses quite different basis and test functions, like for example rooftop functions [29]. FCD has the advantage of being able to exactly represent the function $\Delta \epsilon(\mathbf{r}) \mathbf{E}(\mathbf{r})$ up to the circular frequency $\Delta_{k} / 2$ and also includes the anti-aliasing filtering technique.

\section{RESULTS}

We present in this section 1-D, 2-D, and 3-D calculations. To assess the accuracy of our numerical results, we chose examples where an analytical or an asymptotic solution exists.

The accuracy of CDA results depends on a large number of parameters, in particular on the cell size, the shape, the size and the dielectric constant of the scatterer. Therefore, we restrict this study to glass scatterers $\epsilon_{\mathrm{d}}=2.25$ embedded in vacuum $\epsilon_{\text {ref }}=1$. For each problem a constant geometry is set and we investigate the error as a function of the cell size $\Delta$. More precisely, we report in the figures $\lambda_{\mathrm{d}} / \Delta$, the number of cells per effective wavelength in the dielectric $\lambda_{\mathrm{d}}$. The wavelength in the surrounding medium (vacuum) is $\lambda_{\text {ref }}$.

\section{A. 1-D Calculations}

A 1-D dielectric barrier is embedded in vacuum. The incident wave is a plane wave with propagation direction normal to the barrier. The electric field is parallel to the barrier interfaces. This problem can be solved analytically, thereby providing a perfect reference solution $\mathbf{E}^{\mathrm{a}}(\mathbf{r})$ to assess the accuracy of our procedure [24].

For the numerical results, the barrier is sampled with $N$ samples. We then solve either the associated system of (8) for the Purcell and Pennypacker (PP) solution $\left(M_{i}=0\right)$ or for the traditional coupled-dipole (CDA) solution (nonvanishing $M_{i}$ ) or the system of (26) using the 1-D filtered Green's tensor derived in Appendix A1 for the filtered coupled-dipole (FCD) scheme presented is this paper.

For each of these three methods, the following error function is defined:

$$
\Psi^{1-\mathrm{D}}=\frac{\sum_{i=1}^{N}\left|\mathbf{E}_{i}-\mathbf{E}^{\mathrm{a}}\left(\mathbf{r}_{i}\right)\right|^{2}}{\sum_{i=1}^{N}\left|\mathbf{E}^{\mathrm{a}}\left(\mathbf{r}_{i}\right)\right|^{2}} .
$$

This relative error is represented in Fig. 4 for a barrier length equal to $\lambda_{\text {ref }}$ and $4 \lambda_{\text {ref }}$ and a sampling rate $\lambda_{\mathrm{d}} / \Delta$ between 2 and 30. The number of samples $N$ goes then, respectively, from 3 to 45 points and from 12 to 180 points for the small and large barrier, respectively.

A larger gain in precision between FCD and CDA than between CDA and PP is visible in Fig. 4. This gain is also more important when the barrier is large. This is due the fact that an error is induced throughout the entire barrier length for

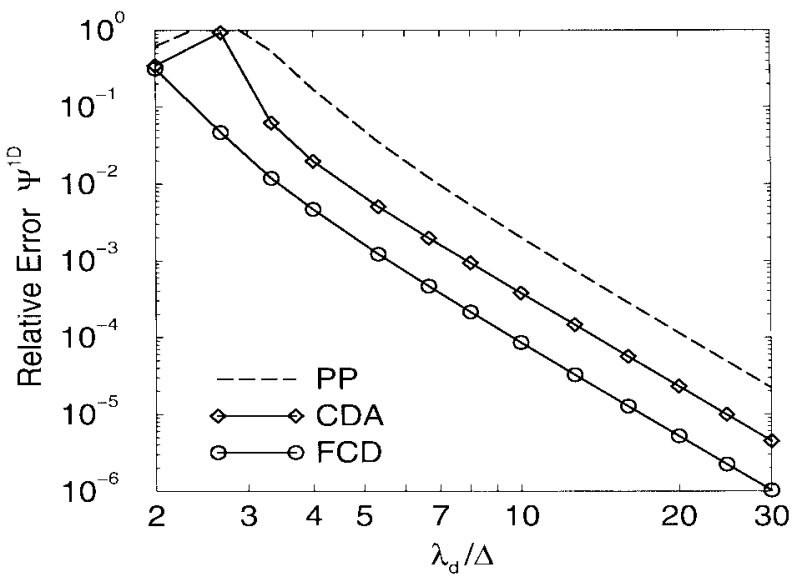

(a)

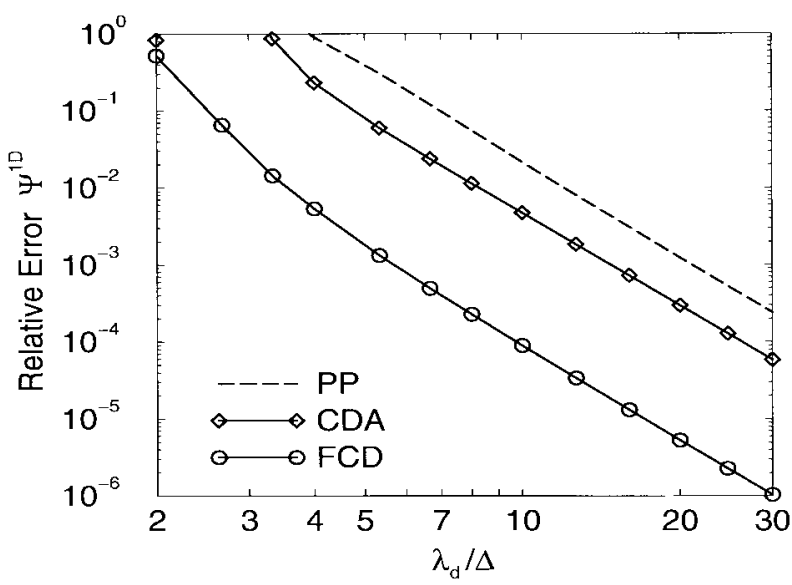

(b)

Fig. 4. Error $\Psi^{1-D}$ for a barrier in vacuum with length $\lambda_{\text {ref }}$ (a), respectively, $4 \lambda_{\text {ref }}$ (b), using the Purcell and Pennypacker scheme (PP), the traditional coupled-dipole approximation (CDA) or the algorithm presented in this paper (FCD). The dielectric barrier has a dielectric constant $\epsilon_{\mathrm{d}}=2.25$.

both PP and CDA schemes, while for FCD an error is only introduced where the function has high spectral components, i.e., at the edges of the barrier, where the dielectric function has a step. In the homogeneous regions, the function can be almost exactly approximated with FCD.

Using PP and CDA it is not possible to calculate an approximation of the field with a large cell size, and the corresponding solutions diverge for less than 3.5, respectively, four samples per wavelength for the small, respectively large barrier. On the other hand, FCD gives a satisfactory field approximation even with a sampling rate close to two points per wavelength (Fig. 4).

\section{B. 2-D Calculations}

A homogeneous dielectric cylinder of radius $R$ is illuminated by a plane wave with a propagation direction perpendicular to the cylinder axis. Two cases can be considered: the electric field of the incident wave parallel to the cylinder axis (TM field) or perpendicular to the axis (TE field). The corresponding Green's tensor and filtered Green's tensor are derived in Appendix A2. The calculation of the latter requires a numerical integration which fortunately converges rapidly. 


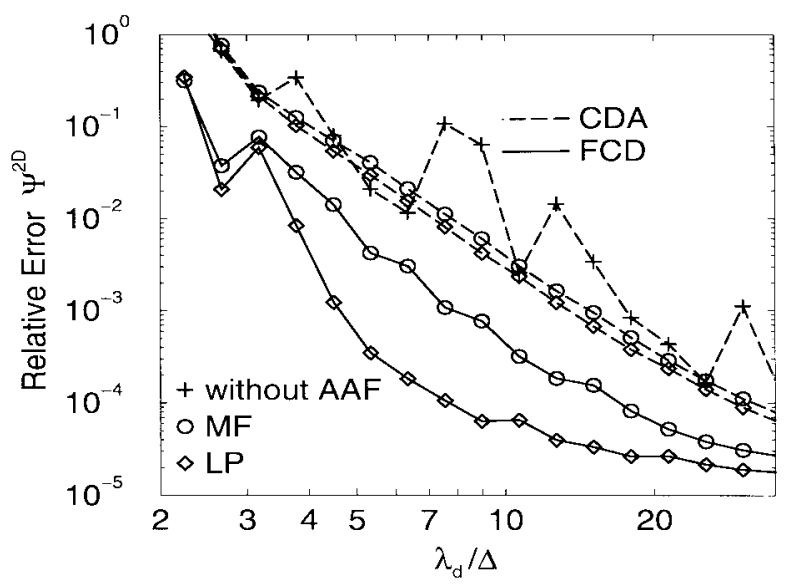

(a)

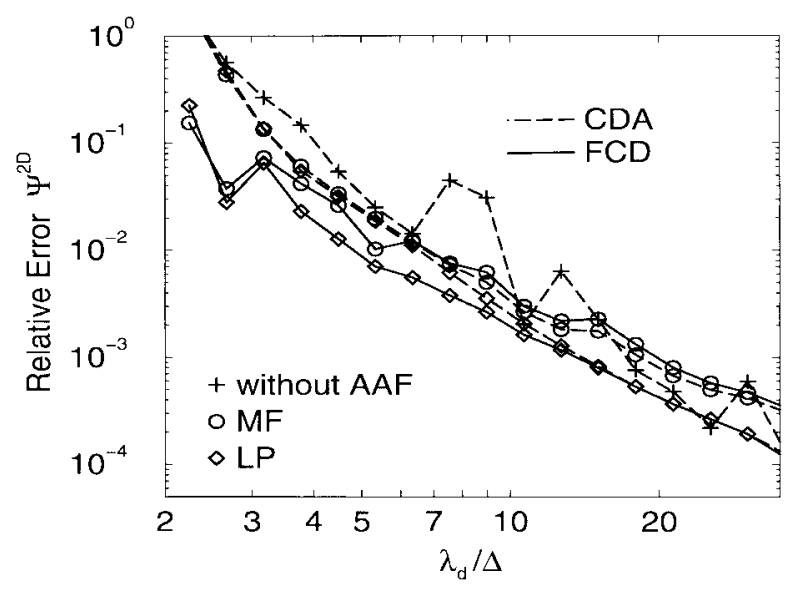

(b)

Fig. 5. Error representation $\Psi^{2-D}$ as a function of the number of cells per effective wavelength $\Delta / \lambda_{\mathrm{d}}$, using the traditional CDA and the algorithm presented in this paper (FCD). The scatterer is a cylinder of radius $R=\lambda_{0}$ in vacuum with dielectric constant $\epsilon_{\mathrm{d}}=2.25$ illuminated, respectively, by (a) a TM field and (b) a TE field. The dielectric function is either directly discretized (without AAF), filtered with a mean-filter (MF) or with a FIR low-pass filter (LP) prior sampling.

As reference solution, we use the asymptotic solution (farfield) $\mathbf{E}_{\mathrm{FF}}^{\mathrm{a}}(\phi)$ scattered in $\phi$-direction [30, Ch. 8]. We then define as error function

$$
\Psi^{2-\mathrm{D}}=\frac{\int_{0}^{2 \pi}\left|\mathbf{E}_{\mathrm{FF}}(\phi)-\mathbf{E}_{\mathrm{FF}}^{\mathrm{a}}(\phi)\right|^{2} \mathrm{~d} \phi}{\int_{0}^{2 \pi}\left|\mathbf{E}_{\mathrm{FF}}^{\mathrm{a}}(\phi)\right|^{2} \mathrm{~d} \phi}
$$

where $\mathbf{E}_{\mathrm{FF}}(\phi)$ is the numerically computed farfield.

The error function $\Psi^{2-D}$ is reported in Fig. 5(a) for the TM case, both for CDA and FCD and for different anti-aliasing filters.

First of all, the curves obtained for CDA without filtering the dielectric function [Fig. 5(a), dashed line with crosses] zigzag substantially. This zigzag effect, which is caused by the more or less good representation of the scatterer geometry by the discretized dipoles, was also observed by Draine [2]. It is strongly attenuated by filtering the dielectric function prior sampling [Fig. 5(a), other dashed lines]. These zigzags cause a large uncertainty on the result accuracy.
The accuracy difference between CDA simulations effectuated either with the mean-filter or with the low-pass filter is small. Therefore the mean-filter, which is easier to implement, seems fully appropriated for CDA.

On the other hand, a large accuracy difference (up to a factor $100)$ is visible between the simulations effectuated with CDA and FCD [Fig. 5(a), dashed and continuous lines]. Furthermore, for the FCD case, filtering with the most sophisticated anti-aliasing filter leads to an accuracy improvement of factor 10, as observed between low-pass (Hanning-FIR) and mean anti-aliasing filters.

FCD simulations effectuated with a low-pass anti-aliasing filter show a rapid convergence for small sampling rate $(5 \Delta \approx$ $\lambda_{\mathrm{d}}$ ), but the convergence decreases for larger sampling rates $\left(20 \Delta \approx \lambda_{\mathrm{d}}\right)$. For such a large sampling rate, FIR anti-aliasing filter seems to have a large influence on the solution accuracy. An optimization of this filter would probably give the possibility to obtain better results, in particular the optimization of the minimization between the linear and nonlinear distortions, and the approximation of the linear filter by a digital one.

Similar behavior is observed for TE polarization [Fig. 5(b)]. In this case, the accuracy improvement is smaller but nevertheless reaches a factor 12 .

Finally the numerical integrations required for the evaluation of the 2-D Filtered Green's tensor increases the computation for FCD. However, this difference represents only a marginal part of the entire computation.

\section{3-D Calculations}

A spherical scatterer of radius $R$ and dielectric constant $\epsilon_{\mathrm{d}}$ is illuminated by a plane wave. The farfield, computed as in [30, Ch. 4], is used again to assess the precision of the numerical results. Defining $\mathbf{E}_{\mathrm{FF}}(\theta, \phi)$ and $\mathbf{E}_{\mathrm{FF}}^{a}(\theta, \phi)$, respectively, as the numerical and the asymptotic farfields radiated in direction $(\theta, \phi)$, the error function becomes

$$
\Psi^{3-\mathrm{D}}=\frac{\int_{0}^{\pi} \int_{0}^{2 \pi}\left|\mathbf{E}_{\mathrm{FF}}(\theta, \phi)-\mathbf{E}_{\mathrm{FF}}^{\mathrm{a}}(\theta, \phi)\right|^{2} \sin (\theta) \mathrm{d} \phi \mathrm{d} \theta}{\int_{0}^{\pi} \int_{0}^{2 \pi}\left|\mathbf{E}_{\mathrm{FF}}^{\mathrm{a}}(\theta, \phi)\right|^{2} \sin (\theta) \mathrm{d} \phi \mathrm{d} \theta} .
$$

This relative error is represented in Fig. 6 as a function of the sampling rate for radii $R=\lambda_{\text {ref }} / 2$ [Fig. 6(a)] and $R=\lambda_{\text {ref }}$ [Fig. 6(b)]. The finest discretization of the larger sphere represents more than 200000 dipoles.

The same considerations as for the 2-D case can be made on the filtering of the dielectric function and on the advantages provided by FCD. The better performances of FCD can be used in two ways: it is possible with FCD to obtain more precision at the same numerical expenses as CDA, or to obtain the same accuracy as CDA for less computation. For example for the sphere $R=\lambda_{\text {ref }}$ with relative error of $10^{-2}$ requires a sampling rate of 3.77, respectively, 6.34 cells per $\lambda_{\mathrm{d}}$ for FCD, respectively, CDA. Such a sampling rate represents 3456 cells for CDA and only 1024 for FCD. On the other hand, with a sampling rate of 6.34 the accuracy is increased by a factor of three between CDA and FCD. 


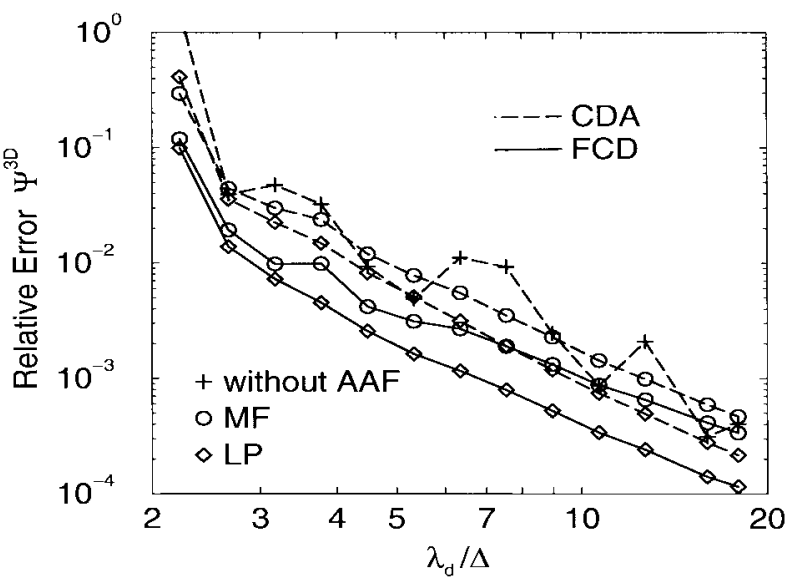

(a)

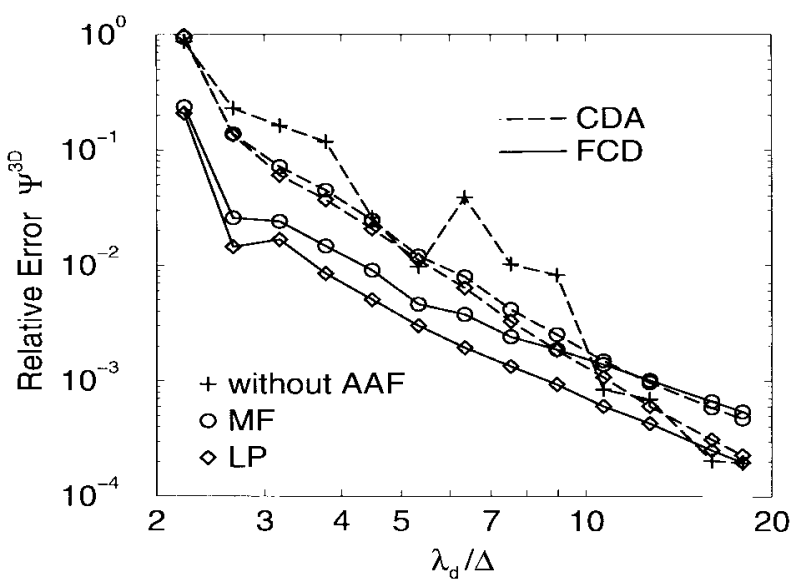

(b)

Fig. 6. Error representation $\Psi^{3-\mathrm{D}}$ as a function of the number of cells per effective wavelength $\Delta / \lambda_{\mathrm{d}}$, using the traditional CDA and the algorithm presented in this paper (FCD). The scatterer is a sphere in vacuum with dielectric constant $\epsilon_{\mathrm{d}}=2.25$ illuminated by a plane wave. Two sphere radii are investigated: (a) $R=0.5 \lambda_{0}$ and (b) $R=\lambda_{0}$. The dielectric function is directly discretized (without AAF), filtered with a mean-filter (MF) or with a FIR low-pass filter (LP) prior sampling.

Because the filtered Green's tensor is known analytically for 3-D (Appendix A3) and therefore no numerical integration is necessary for its calculation, the time required to evaluate the Green's tensors merely differs between CDA and FCD. Furthermore, it is totally negligible compared to the total computation time.

We have also tested these different schemes thoroughly with other dielectric constants $\epsilon_{\text {ref }}$. While a small $\epsilon_{\text {ref }}$ provides very good convergence and accuracy, a finer discretization is required for larger dielectric constants or for absorbing media. The accuracy improvements provided by FCD and AAF where approximately identical for each case.

The solution of the system of equations was obtained with a conjugate gradient code (CG) made available to the public domain by Flatau et al. [31]. The matrix products required by CG were computed by FFT using the NAG library [32]. The symmetry of the problem was taken into account to reduce the size of the system of equations. The condition of the associated matrix is not sensibly influenced by the filtering of Green's tensor, the number of iterations of the CG being then quite similar for CDA and FCD.

\section{CONCLUSIONS}

A new numerical scheme, the filtered coupled-dipole (FCD), based on the sampling theory, has been developed for 1-D, 2-D, and 3-D.

FCD increases the performance of the coupled-dipole approximation (CDA): with this new scheme it is possible to obtain with a given discretization grid a better accuracy than with CDA; or to achieve the same accuracy with a larger cell size. The additional computation time is negligible for 1-D and 3-D systems; for 2-D systems it is below $10 \%$ of the total computation time.

This new scheme is particularly well-suited for large scatterers with homogeneous subdomains. Furthermore, while CDA results diverge for low discretization rates, FCD continues to provide reasonable results with a discretization rate close to 2 points per effective wavelength.

Filtering of the dielectric function $\Delta \epsilon(\mathrm{r})$ prior to sampling has been shown to have a strong influence on the accuracy of the results. In particular, the use of a mean-filter [which corresponds to averaging $\Delta \epsilon(\mathbf{r})$ over each cell] also improves the results of standard CDA. More sophisticated filters were investigated and, the best results were obtained with a lowpass filter. A detailed study of the influence of the filter characteristic on the accuracy of the method remains to be done.

\section{APPENDIX}

\section{A. Derivation of the Filtered Free-Space Green's Tensor}

In this section, the filtered Green's Tensor $\stackrel{\leftrightarrow}{G}^{\mathrm{F}}\left(\mathbf{r}, \mathbf{r}^{\prime}\right)$ for free space is derived. We first explain the transformation of (25) into a convolution, which makes its computation in the spectral domain possible. Then this scheme is used to derivate 1-D, 2-D, and 3-D filtered Green's tensors.

For free space, the Green's dyadic $\overleftrightarrow{G}\left(\mathbf{r}, \mathbf{r}^{\prime}\right)$ is only a function of the relative position of $\mathbf{r}^{\prime}$ and $\mathbf{r}$ [33, Sec. 4.2]. Therefore, it is possible to rewrite this dyadic as a function of the relative position only:

$$
\overleftrightarrow{G}\left(\mathbf{r}, \mathbf{r}^{\prime}\right)=\overleftrightarrow{G}\left(\mathbf{r}-\mathbf{r}^{\prime}\right) .
$$

Equation (30) defines the single argument Green's tensor $\overleftrightarrow{G}\left(\mathbf{r}-\mathbf{r}^{\prime}\right)$. With this notation, (25) becomes, after replacing the integration variable $\mathbf{r}^{\prime}$ by $\mathrm{r}^{\prime \prime}=\mathbf{r}^{\prime}-\mathrm{r}_{k}$,

$$
\overleftrightarrow{G}^{\mathrm{F}}\left(\mathbf{r}, \mathbf{r}_{k}\right)=\lim _{\delta V^{\prime \prime} \rightarrow 0} \int_{V^{\prime \prime}-\delta V^{\prime \prime}} \overleftrightarrow{G}\left(\mathbf{r}-\mathbf{r}_{k}-\mathbf{r}^{\prime \prime}\right) h^{\mathrm{r}}\left(\mathbf{r}^{\prime \prime}\right) \mathrm{d} \mathbf{r}^{\prime \prime}
$$

where the integration volume $V^{\prime \prime}-\delta V^{\prime \prime}$ represents the initial integration volume shifted by the change of integration variable. We define a new tensor $\vec{G}^{\prime}(\mathbf{r})$ that is identical to $\overleftrightarrow{G}(\mathbf{r})$ except in the exclusion domain, where it vanishes:

$$
\overleftrightarrow{G}^{\prime}(\mathbf{r})= \begin{cases}\overleftrightarrow{0} & \text { if } \mathrm{r} \in \delta V^{\prime \prime} \\ \stackrel{G}{G}(\mathrm{r}) & \text { elsewhere }\end{cases}
$$

In Section II, it was mentioned that $\Delta \epsilon(\mathbf{r})$ vanishes outside $V^{\prime \prime}$; as $\Delta \epsilon(\mathbf{r})$ enters in $h^{\mathrm{r}}\left(\mathbf{r}^{\prime \prime}\right)$, it is possible to expand the 
integration volume $V^{\prime \prime}$ to the infinite domain $\mathbb{R}^{D}$, where $D$ is the number of dimensions of the problem. Extending the integration domain to $\mathbb{R}^{D}$ and replacing the Green's tensor of (31) with $\overleftrightarrow{G}^{\prime}(\mathbf{r})$ leads to

$$
\overleftrightarrow{G}^{\mathrm{F}}\left(\mathbf{r}, \mathbf{r}_{k}\right)=\lim _{\delta V^{\prime \prime} \rightarrow 0} \int_{\mathbf{R}^{D}} \overleftrightarrow{G}^{\prime}\left(\mathbf{r}-\mathrm{r}_{k}-\mathrm{r}^{\prime \prime}\right) h^{\mathrm{r}}\left(\mathbf{r}^{\prime \prime}\right) \mathrm{dr}^{\prime \prime} .
$$

Equation (33) is the convolution of $h^{\mathrm{r}}(\mathbf{r})$ with $\overleftrightarrow{G}^{\prime}(\mathbf{r})$ at the point $\mathbf{r}-\mathbf{r}_{k}$. A convenient way to evaluate a convolution is to use the spectral domain, where the spectrum of the convolution of two functions becomes the product of their spectra. We define $\overleftrightarrow{\Gamma}^{\prime}(\mathbf{k})$ as the spectrum of $\overleftrightarrow{G}^{\prime}(\mathbf{r})$ and $H^{\mathrm{r}}(\mathbf{k})$ as the spectrum of $h^{\mathrm{r}}(\mathbf{r})$. Using the inverse Fourier transform of the product of these two spectra, the filtered Green's tensor becomes

$$
\stackrel{G}{G}^{\mathrm{F}}\left(\mathbf{r}, \mathbf{r}_{k}\right)=\frac{1}{(2 \pi)^{D}} \int_{\mathbf{R}^{D}} \stackrel{\leftrightarrow}{\Gamma}^{\prime}(\mathbf{k}) \mathrm{e}^{i \mathbf{k}\left(\mathbf{r}-\mathbf{r}_{k}\right)} H^{\mathrm{r}}(\mathbf{k}) \mathrm{d} \mathbf{k} .
$$

If the reconstruction filter $H^{\mathrm{r}}(\mathbf{k})$ has a pass-through domain $\mathcal{D}_{\text {PT }}$ where its spectrum is equal to 1 and a blocking domain $\mathcal{D}_{\mathrm{B}}$ where its spectrum vanishes, the integration domain in (34) can be restricted to $\mathcal{D}_{\mathrm{PT}}$ :

$$
\stackrel{\leftrightarrow}{G}^{\mathrm{F}}\left(\mathbf{r}, \mathbf{r}_{k}\right)=\frac{1}{(2 \pi)^{D}} \int_{\mathcal{D}_{\mathrm{PT}}} \stackrel{\leftrightarrow}{\Gamma}^{\prime}(\mathbf{k}) \mathrm{e}^{i \mathbf{k}\left(\mathbf{r}-\mathbf{r}_{k}\right)} \mathrm{dk}
$$

In our case, the pass-through domain of the filter $\mathcal{D}_{\mathrm{PT}}$ is a closed domain. The nonvanishing spectral part of the filtered Green's tensor is also included in a closed domain. Functions having such a spectrum do not have any singularity. Therefore, contrary to the Green's tensor $\overleftrightarrow{G}\left(\mathrm{r}, \mathbf{r}^{\prime}\right)$, the filtered Green's tensor does not have a singularity for $\mathbf{r}=\mathrm{r}^{\prime}$.

The filtered Green's tensor can also be evaluated as the difference between the Green's tensor and the Green's tensor filtered by the complementary filter (a high-pass with passthrough domain $\mathcal{D}_{\mathrm{B}}$ ). For $\mathbf{r} \neq \mathbf{r}_{k}$, this leads to

$$
\overleftrightarrow{G}^{\mathrm{F}}\left(\mathbf{r}, \mathbf{r}_{k}\right)=\overleftrightarrow{G}\left(\mathbf{r}, \mathbf{r}_{k}\right)-\frac{1}{(2 \pi)^{D}} \int_{\mathcal{D}_{\mathrm{B}}} \overleftrightarrow{\Gamma}^{\prime}(\mathbf{k}) \mathrm{e}^{i \mathbf{k}\left(\mathbf{r}-\mathbf{r}_{k}\right)} \mathrm{d} \mathbf{k} .
$$

Both terms of the right side of (36) have a singularity for $\mathbf{r}=\mathrm{r}_{k}$. These two singularities compensate each other to give the regular function (35) without any singularity. It is therefore possible to evaluate the filtered Green's tensor for $\mathbf{r}=\mathrm{r}_{k}$ with a limit calculation $\mathrm{r}^{\prime} \rightarrow \mathrm{r}$ on (36).

1) 1-D Green's Tensor: For the propagation direction parallel to the $\hat{x}$ axis, the free-space 1-D dyadic Green's tensor is

$$
\overleftrightarrow{G}\left(x-x^{\prime}\right)=\frac{i}{2 k_{\text {ref }}} \mathrm{e}^{i k_{\text {ref }}\left|x-x^{\prime}\right| \overleftrightarrow{I}_{\mathrm{t}}}
$$

where $k_{\text {ref }}=\sqrt{\epsilon_{\text {ref }}} k_{0}$ is the wavenumber of the reference medium and $\overleftrightarrow{I}_{\mathrm{t}}$ the transversal unit dyadic defined as $\overleftrightarrow{I}_{\mathrm{t}}=$ $\hat{y} \hat{y}+\hat{z} \hat{z}$. Evaluating the Fourier transform of the single argument Green's dyadic gives

$$
\overleftrightarrow{\Gamma}^{\prime}(k)=\frac{1}{k-k_{\text {ref }}} \stackrel{\leftrightarrow}{\mathrm{t}}_{\mathrm{f}}
$$

The spectrum $\overleftrightarrow{\Gamma}^{\prime}(k)$ has two poles for $k= \pm k_{\text {ref }}$. These poles falling in the pass-through domain of the filter (a low-pass with circular frequency $k_{\mathrm{F}}=\Delta_{k} / 2$ ), the filtered Green's tensor is derived using (36). This gives for $x \neq x^{\prime}$

$$
\begin{aligned}
\overleftrightarrow{G}^{F}\left(x, x^{\prime}\right)= & \stackrel{\leftrightarrow}{G}\left(x, x^{\prime}\right)+\cos \left(k_{\mathrm{ref}} l\right) \frac{C^{-}(l)-C^{+}(l)}{2 \pi k_{\text {ref }}} \overleftrightarrow{I}_{\mathrm{t}} \\
& +\sin \left(k_{\mathrm{ref}} l\right) \frac{\pi-S^{+}(l)-S^{-}(l)}{2 \pi k_{\text {ref }}} \overleftrightarrow{I}_{\mathrm{t}}
\end{aligned}
$$

where

$$
\begin{aligned}
& C^{ \pm}(l)=\operatorname{Ci}\left(\left(k_{\mathrm{F}} \pm k_{\mathrm{ref}}\right) l\right) \\
& S^{ \pm}(l)=\operatorname{Si}\left(\left(k_{\mathrm{F}} \pm k_{\mathrm{ref}}\right) l\right)
\end{aligned}
$$

and $l=\left|x-x^{\prime}\right|$. The limit calculation $x \rightarrow x^{\prime}$ must then be used to evaluate the Green's dyadic on its pseudo-singularity $x=x^{\prime}$.

2) 2-D Green's Tensor: Assuming that the 2-D problem has a translation symmetry along the $\hat{z}$ direction and that the field propagates only in the $\hat{x}, \hat{y}$ plane (i.e., that there is no coupling between TM and TE fields), the associated Green's tensor is

$$
\overleftrightarrow{G}\left(\mathbf{r}-\mathbf{r}^{\prime}\right)=\left[\begin{array}{ccc}
\frac{1}{2} a+\frac{1}{2} c & \frac{1}{2} b & 0 \\
\frac{1}{2} b & \frac{1}{2} a-\frac{1}{2} c & 0 \\
0 & 0 & a
\end{array}\right]
$$

where $a, b$, and $c$ are functions of $\mathbf{r}-\mathrm{r}^{\prime}$. Defining $\phi$ and $\rho$ as argument, respectively, length of the vector $\mathbf{r}-\mathbf{r}^{\prime}$, these parameters are

$$
\begin{aligned}
a & =\frac{i}{4} \mathrm{H}_{0}^{(1)}\left(k_{\mathrm{ref}} \rho\right) \\
b & =\frac{i}{4} \mathrm{H}_{2}^{(1)}\left(k_{\mathrm{ref}} \rho\right) \sin (2 \phi) \\
c & =\frac{i}{4} \mathrm{H}_{2}^{(1)}\left(k_{\mathrm{ref}} \rho\right) \cos (2 \phi) .
\end{aligned}
$$

We evaluate the Fourier transform of each parameter $a, b$, and $c$, excluding a circular volume $\delta V$ from the integral. Taking advantage of the radial dependence of the parameters, their Fourier transform can be performed using the Hankel transform [33, Sec. 1.4]. This gives

$$
\begin{aligned}
& \mathcal{F}\left\{a^{\prime}\right\}=\frac{1}{k^{2}-k_{\text {ref }}^{2}}, \\
& \mathcal{F}\left\{b^{\prime}\right\}=\left(\frac{k}{k_{\text {ref }}}\right)^{2} \frac{-1}{k^{2}-k_{\text {ref }}^{2}} \cos (2 \theta) \\
& \mathcal{F}\left\{c^{\prime}\right\}=\left(\frac{k}{k_{\text {ref }}}\right)^{2} \frac{-1}{k^{2}-k_{\text {ref }}^{2}} \sin (2 \theta)
\end{aligned}
$$

where $\mathcal{F}\{\}$ represents the Fourier transform and, in an analog notation as (32), the prime has been affixed to $a, b$, and $c$ because the Fourier transform is evaluated by integration on the entire domain except the exclusion volume $\delta V$. The argument and length of the vector $\mathbf{k}$ are $\theta$, respectively, $k$.

The spectra (46)-(48) can be expressed with the function product $S(k) \xi(n \theta)$, where $\xi()$ is one of the functions $\sin ()$ or $\cos ($ ), and $S(k)$ the remaining part of the function, which is only dependent on $k$. As the function $S(k)$ has poles lying near the pass-through domain $\mathcal{D}_{\mathrm{PT}}$ of the filter, (36) is again 


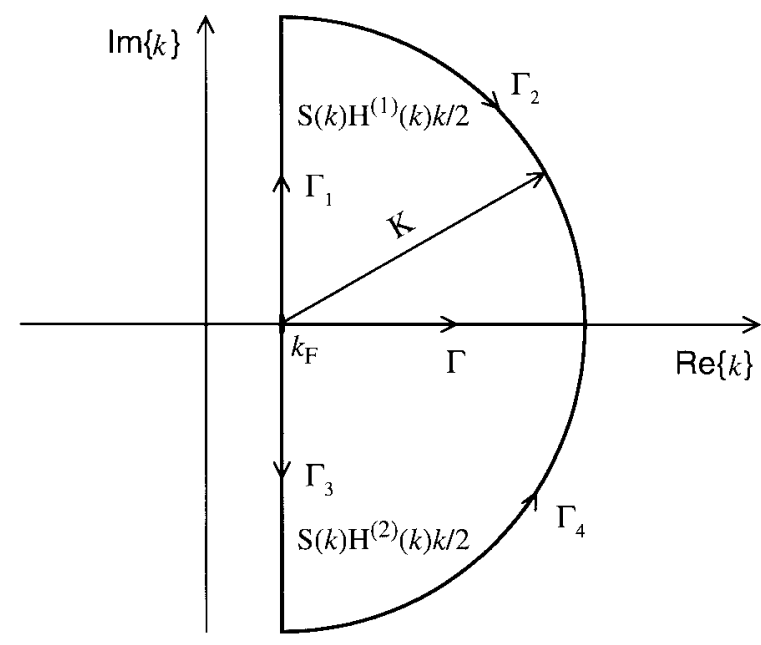

Fig. 7. Integration path for the numerical integration of the 2-D filtered Green's function.

the most appropriate to compute the filtered parameters. The pass-through domain of the filter being a circle of radius $k_{\mathrm{F}}$, the filtered parameters gives for $r \neq \mathbf{r}^{\prime}$, after integration of the azimuthal component

$$
f^{\mathrm{F}}=f-\frac{i^{n} \xi(n \phi)}{2 \pi} \int_{k_{\mathrm{F}}}^{\infty} S(k) \mathrm{J}_{n}(k \rho) k \mathrm{~d} k
$$

where $f$ is one of the $a, b$, or $c$ parameters. Again, for $\mathbf{r}=\mathbf{r}^{\prime}$, the limit $\mathrm{r} \rightarrow \mathrm{r}^{\prime}$ must be evaluated.

The evaluation of the filtered coefficients requires a numerical integration from $k_{\mathrm{F}}$ to infinity. The function to integrate contains a Bessel function, which means an oscillating slow convergence. To improve the convergence, we decompose the integral into two by expanding the Bessel function into a sum of Hankel functions of the first and second kinds $\mathrm{J}_{n}(z \rho)=\mathrm{H}_{n}^{(1)}(z \rho) / 2+\mathrm{H}_{n}^{(2)}(z \rho) / 2$ and choosing an appropriate integration path in the complex plane for each of these two integrals (Fig. 7). Indeed, because the two integrand are analytic and have no singular points for real values greater or equal to $k_{\mathrm{F}}$, the modification of the integration paths from $\Gamma$ to $\Gamma_{1}+\Gamma_{2}$ or from $\Gamma$ to $\Gamma_{3}+\Gamma_{4}$ does not change the result of the integral. Giving the integration path $\Gamma_{1}+\Gamma_{2}$ and $\Gamma_{3}+\Gamma_{4}$, respectively, for the integral with the first and second Hankel functions, respectively, and making the radius $K$ go to infinity, the integration path $\Gamma$ corresponds exactly to the integration path of (49) and the integrand vanishes on $\Gamma_{2}$ and $\Gamma_{4}$. The two remaining integrals converge very rapidly because the Hankel function with a complex argument does not oscillate and decreases extremely rapidly.

3) 3-D Green's Tensor: The 3-D free space Green's Tensor $\overleftrightarrow{G}\left(\mathbf{r}-\mathbf{r}^{\prime}\right)$ can be expressed, for $\mathbf{r} \neq \mathbf{r}^{\prime}$, by the differentiation of the scalar Green's function $g\left(\left|\mathbf{r}-\mathbf{r}^{\prime}\right|\right)$

$$
\overleftrightarrow{G}\left(\mathbf{r}-\mathbf{r}^{\prime}\right)=\left(\stackrel{\leftrightarrow}{I}+\frac{1}{k_{\text {ref }}^{2}} \nabla \nabla\right) g\left(\left|\mathbf{r}-\mathbf{r}^{\prime}\right|\right)
$$

where the scalar Green's function is

$$
g\left(\left|\mathrm{r}-\mathrm{r}^{\prime}\right|\right)=\frac{\mathrm{e}^{i k_{\mathrm{ref}} R}}{4 \pi R}
$$

with $R=\left|\mathbf{r}-\mathbf{r}^{\prime}\right|$. Introducing the Green's tensor definition (50) into the integral of the filtered Green's tensor (33) gives after interchange of the integration/differentiation order

$$
\begin{aligned}
\overleftrightarrow{G}^{\mathrm{F}}\left(\mathbf{r}, \mathrm{r}_{k}\right)= & \left(\overleftrightarrow{I}+\frac{1}{k_{\text {ref }}^{2}} \nabla \nabla\right) \lim _{\delta V^{\prime \prime} \rightarrow 0} \int_{V^{\prime \prime}-\delta V^{\prime \prime}} \\
& \cdot g\left(\left|\mathbf{r}-\mathbf{r}_{k}-\mathbf{r}^{\prime \prime}\right|\right) h^{\mathrm{r}}\left(\mathbf{r}^{\prime \prime}\right) \mathrm{d} \mathbf{r}^{\prime \prime}+\frac{\overleftrightarrow{I}}{3 k_{\text {ref }}^{2}} h^{\mathrm{r}}\left(\mathbf{r}-\mathbf{r}_{k}\right)
\end{aligned}
$$

where a spherical shape of the exclusion volume has been assumed. We notice the apparition outside the integral of a term that only depends on the impulse response $h^{\mathrm{r}}\left(\mathbf{r}-\mathrm{r}_{k}\right)$. This term, which is similar to the term $\overleftrightarrow{L}$ in (4) for a spherical exclusion volume, is caused by the differentiation/integration order exchange [34, eq. (4.14)].

The integration in (52) being a convolution, its result represents the filtered scalar Green's function, which we define by $g^{\mathrm{F}}\left(\left|\mathbf{r}-\mathbf{r}_{k}\right|\right)$. As for the 1-D and 2-D cases, the spectrum is used to filter this function. The Fourier transform of the Green's function is evaluated using the Hankel transform [35, p. 254]:

$$
\mathcal{F}\left\{g^{\prime}(r)\right\}=\frac{1}{k^{2}-k_{\text {ref }}^{2}}
$$

where the exclusion volume $\delta V$ has a spherical shape to comply with the shape assumed for the derivation/integration interchange in (52).

According to (36), the filtered Green's function can be determined by subtracting from the Green's function the Green's function filtered with the complementary filter. In our case, the Green's function filtered with a low-pass filter with a spherical pass-through domain of radius $k_{\mathrm{F}}$ can be evaluated by inverse Hankel transform. This gives for $r \neq 0$

$$
\begin{aligned}
g^{\mathrm{F}}(r)= & g(r)-\cos \left(k_{\mathrm{ref}} r\right) \frac{C^{-}(r)-C^{+}(r)}{(2 \pi)^{2} r} \\
& -\sin \left(k_{\mathrm{ref}} r\right) \frac{\pi-S^{+}(r)-S^{-}(r)}{(2 \pi)^{2} r}
\end{aligned}
$$

where the functions $C^{ \pm}(r)$ and $S^{ \pm}(r)$ are defined in (40) and (41). Introducing the filtered Green's function into the filtered Green's tensor (52) leads to

$$
\begin{aligned}
\stackrel{\leftrightarrow}{G}^{\mathrm{F}}\left(\mathbf{r}, \mathbf{r}_{k}\right)= & \left(\overleftrightarrow{I}+\frac{1}{k_{\text {ref }}^{2}} \nabla \nabla\right) g^{\mathrm{F}}\left(\left|\mathbf{r}-\mathbf{r}_{k}\right|\right) \\
& +\frac{\overleftrightarrow{I}}{3 k_{\text {ref }}^{2}} h^{\mathrm{r}}\left(\mathbf{r}-\mathbf{r}_{k}\right) .
\end{aligned}
$$

Because $g^{\mathrm{F}}(R)$ is known analytically, the application of the operator $\left(\stackrel{\leftrightarrow}{I}+1 / k_{\text {ref }}^{2} \nabla \nabla\right)$ to the filtered Green's function can be determined without difficulties. Only the filter impulse response in (55) must still be determined. The function $H^{\mathrm{r}}(\mathbf{k})$ being equal to one in a sphere of radius $k_{\mathrm{F}}$ and zero elsewhere, its inverse Fourier transform gives

$$
h^{\mathrm{r}}(\mathbf{r})=\frac{\sin \left(k_{\mathrm{F}}|\mathbf{r}|\right)-k_{\mathrm{F}}|\mathbf{r}| \cos \left(k_{\mathrm{F}}|\mathbf{r}|\right)}{2 \pi^{2}|\mathbf{r}|^{3}} .
$$


The filtered Green's function $\overleftrightarrow{G}^{\mathrm{F}}\left(\mathbf{r}, \mathbf{r}_{k}\right)$ is now fully determined for $\mathbf{r} \neq \mathbf{r}_{k}$. A similar limit calculation as in the 1-D and 2-D cases must be effectuated for $\mathbf{r}=\mathbf{r}_{k}$,

$$
\stackrel{\leftrightarrow}{G}^{\mathrm{F}}\left(\mathbf{r}_{k}, \mathbf{r}_{k}\right)=\lim _{\mathbf{r} \rightarrow \mathbf{r}_{k}} \stackrel{\leftrightarrow}{G}^{\mathrm{F}}\left(\mathbf{r}, \mathbf{r}_{k}\right) \text {. }
$$

\section{ACKNOWLEDGMENT}

N. B. Piller is most indebted to O. Johnsen who introduced him to the power and beauty of the signal processing. It is a pleasure to acknowledge fruitful discussions with all our colleagues, notably R. Ballisti, C. Hafner, P. Leuchtmann, and R. Vahldieck. The authors are thankful to P. J. Flatau et al. for making their excellent $\mathrm{CG}$ code available to the public domain [31].

\section{REFERENCES}

[1] E. M. Purcell and C. R. Pennypacker, "Scattering and absorption of light by nonspherical dielectric grains," Astrophys. J., vol. 186, pp. 705-714, Dec. 1973.

[2] B. T. Draine, "The discrete-dipole approximation and its applications to interstellar graphite grains," Astrophys. J., vol. 333, pp. 848-872, Oct. 1988

[3] G. H. Goedecke and S. O'Brien, "Scattering by irregular inhomogeneous particles via the digitized Green's function algorithm," Appl. Opt., vol. 27, no. 12, pp. 2431-2438, June 1988.

[4] W. T. Doyle, "Optical properties of a suspension of metal spheres," Phys. Rev. B, vol. 39, no. 14, pp. 9852-9858, May 1989.

[5] V. V. Varadan, A. Lakhtakia, and V. K. Varadan, "Scattering by threedimensional anisotropic scatterers," IEEE Trans. Antennas Propagat. vol. 37, pp. 800-802, June 1989.

[6] A. Llakhtakia, "General theory of the Purcell-Pennypacker scattering approach and its extension to bianisotropic scatterers," Astrophys. J., vol. 394, pp. 494-499, Aug. 1992.

[7] O. J. F. Martin, A. Dereux, and C. Girard, "An iterative scheme to compute exactly the total field propagating in dielectric structures of arbitrary shapes," J. Opt. Soc. Amer. A, vol. 11, pp. 1073-1080, 1994.

[8] J. J. Goodman, B. T. Draine, and P. T. Flatau, "Application of the fastFourier-transformation techniques to the discrete-dipole approximation," Opt. Lett., vol. 16, no. 15, pp. 1198-1200, Aug. 1991.

[9] B. T. Draine and P. J. Flatau, "Discrete-dipole approximation for scattering calculations," J. Opt. Soc. Amer. A, vol. 11, no. 4, pp. 1491-1499, Apr. 1994

[10] M. Petravic and G. Kuo-Petravic, "An ILUCG algorithm which minimizes in the Euclidean norm," J. Comput. Phys., vol. 32, pp. 263-269, 1979.

[11] A. Lakhtakia, "Strong and weak forms of the method of moments and the coupled dipole method for scattering of time-harmonic electromagnetic fields," Int. J. Mod. Phys. C, vol. 3, no. 3, pp. 583-603, Mar. 1992.

[12] J. Rahola, "Solution of dense systems of linear equations in the discretedipole approximation," SIAM J. Sci. Comput., vol. 17, pp. 78-89, 1996.

[13] W. C. Chew, C. C. Lu, and Y. M. Wand, "Efficient computation of three-dimentional scattering of vector electromangetic waves," J. Opt. Soc. Amer., vol. 11, no. 4, p. 1528, Apr. 1994.

[14] A. D. Yaghiian, "Electric dyadic Green's functions in the source region," Proc. IEEE, vol. 68, pp. 248-263, Feb. 1980.

[15] J. G. Fikioris, "Electromagnetic field inside a current-carrying region," J. Math. Phy, vol. 6, no. 11, p. 1617, Nov. 1965.

[16] J. C. Ku, "Comparisons of coupled-dipole solutions and dipole refractive indices for light scattering and absorptions by arbitrarily shaped ar agglomerated particles," J. Opt. Soc. Amer., vol. 10, no. 2, pp. 336-342, Feb. 1993.

[17] M. A. Taubenblatt and T. K. Tran, "Calculation of light scattering from particles and structures on a surface by the coupled-dipole method," $J$. Opt. Soc. Amer. A, vol. 10, no. 5, pp. 912-919, May 1993.

[18] J. J. Spilker, "Theoretical bounds on the performance of sampled data communications systems," IRE Trans. Circuit Theory, vol. 7, pp. 335-341, Sept. 1960

[19] W. E. Brown, "Optimum prefiltering of sampled data," IRE Trans. Inform. Theory, vol. 7, pp. 269-270, Oct. 1961.
[20] N. Mohanty, Signal Processing: Signals, Filtering and Detection. New York: Van Nostrand Reinhold, 1987, ch. 1.5.

[21] B. M. Oliver, J. R. Pierce, and C. E. Shannon, "The philosophy of PCM," Proc. IRE, vol. 36, no. 234, pp. 1324-1331, Nov. 1948.

[22] C. E. Shannon, "Communication in the presence of noise," Proc. IRE, vol. 37, pp. 10-21, Jan. 1949.

[23] D. P. Petersen and D. Middleton, "Sampling and reconstruction of wavenumber-limited functions in $n$-dimensional Euclidean spaces," Inform. Contr., vol. 5, pp. 279-322, 1962

[24] C. Cohen-Tannoudji, B. Diu, and F. Laloe, Quantum Mechanics. Paris, France: Hermann, 1977.

[25] C. Hafner and L. Bomholt, The 3D Electromagnetic Wave Simulator. Chichester, U.K.: Wiley, 1993.

[26] I. N. Vekua, "New methods for solving elliptic equations," in Applied Mathematics and Mechanics. Amsterdam, The Netherlands: NorthHolland, 1967, vol. 1.

[27] B. Moon and D. E. Spencer, Field Theory Handbook, 2nd ed. Berlin, Germany: Springer-Verlag, 1971.

[28] L. R. Rabiner and B. Gold, Theory and Application of Digital Signal Processing. Englewood Cliffs, NJ: Prentice-Hall, 1975.

[29] H. Gan and W. C. Chew, "A discrete BCCG-FFT algorithm for solving 3-D inhomogeneous scatterer problems," J. Electromagn. Waves Applicat., vol. 9, no. 10, p. 1339, 1995

[30] C. F. Bohren and D. R. Huffman, Absorption and Scattering of Light by Small Particles. New York: IEEE Press, 1980.

[31] P. J. Flatau, T. Schneider, and F. Evans, CCGPAK. Institution of Oceanography [Online]. University of California. Available www: http://atol.ucsd.edu/ p platau/scatlib/conjugate.html

[32] The Numerical Algorithms Group. Oxford, U.K.: NAG Ltd. Available www: http://www.nag.co.uk

[33] C.-T. Tai, Dyadic Green Functions in Electromagnetic Theory, 2nd ed. New York: IEEE Press, 1993.

[34] J. Van Kranendonk and J. E. Sipe, "Foundations of the macroscopic electromagnetic theory of dielectric media," in Progress in Optics. Amsterdam, The Netherlands: North-Holland, 1977, vol. 15, ch. 5, pp. 245-350.

[35] R. N. Barcewell, The Fourier Transform and Its Applications, 2nd ed. New York: McGraw-Hill, 1978

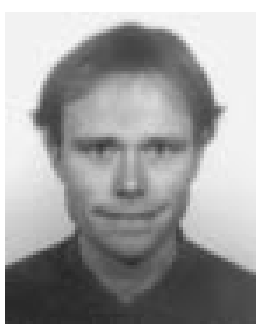

Nicolas B. Piller (S'98) received the Dipl.Ing. ETS degree from Ecole d'Ingénieurs de Fribourg, Fribourg, Switzerland, in 1991 and the Electrical Engineering degree from the Swiss Federal Institute of Technology (ETH) Zurich, Switzerland, in 1995. He is currently working toward the Ph.D. degree from the same university.

In April 1995, he joined the Laboratory of Field Theory and Microwave Electronics of the Swiss Federal Institute of Technology (ETH) Zurich. His research interests include the development of computational techniques for optics and the investigation of the interaction of light with anisotropic media.

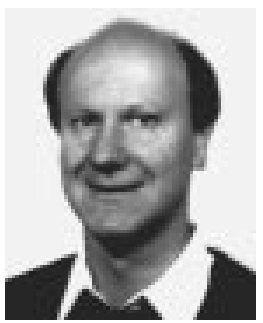

Olivier J. F. Martin received the B.S. and Ph.D. degrees in physics in 1989 and 1994, respectively, from the Swiss Federal Institute of Technology, Lausanne (EPFL), Switzerland.

In 1989, he joined IBM Zurich Research Laboratory, where he investigated the thermal and optical properties of semiconductor laser diodes. Since 1994 he has been a Research Staff Member at the Swiss Federal Institute of Technology Zurich $($ ETH). Since 1998 he has been a Lecturer at ETH His current research interests are focused on the analysis of the interaction of electromagnetic fields with mesoscopic systems, with an emphasis on local probe microscopes (scanning tunneling microscope, scanning near-field optical microscope).

Dr. Martin received a special tenure Fellowship from the Swiss National Science Foundation in 1997. 